\title{
High-aspect ratio metal tips attached to atomic force microscopy cantilevers with controlled angle, length, and radius for electrostatic force microscopy
}

Cite as: Rev. Sci. Instrum. 78, 113706 (2007); https://doi.org/10.1063/1.2805513

Submitted: 09 August 2007. Accepted: 15 October 2007. Published Online: 09 November 2007

Lynda Cockins, Yoichi Miyahara, Romain Stomp, and Peter Grutter

ARTICLES YOU MAY BE INTERESTED IN

Scanning probe tip geometry optimized for metrology by focused ion beam ion milling Journal of Vacuum Science \& Technology B: Microelectronics and Nanometer Structures Processing, Measurement, and Phenomena 9, 3569 (1991); https://doi.org/10.1116/1.585846

Development of a metal-tip cantilever for noncontact atomic force microscopy

Review of Scientific Instruments 76, 033705 (2005); https://doi.org/10.1063/1.1865812

Frequency modulation detection using high-Q cantilevers for enhanced force microscope sensitivity

Journal of Applied Physics 69, 668 (1991); https://doi.org/10.1063/1.347347

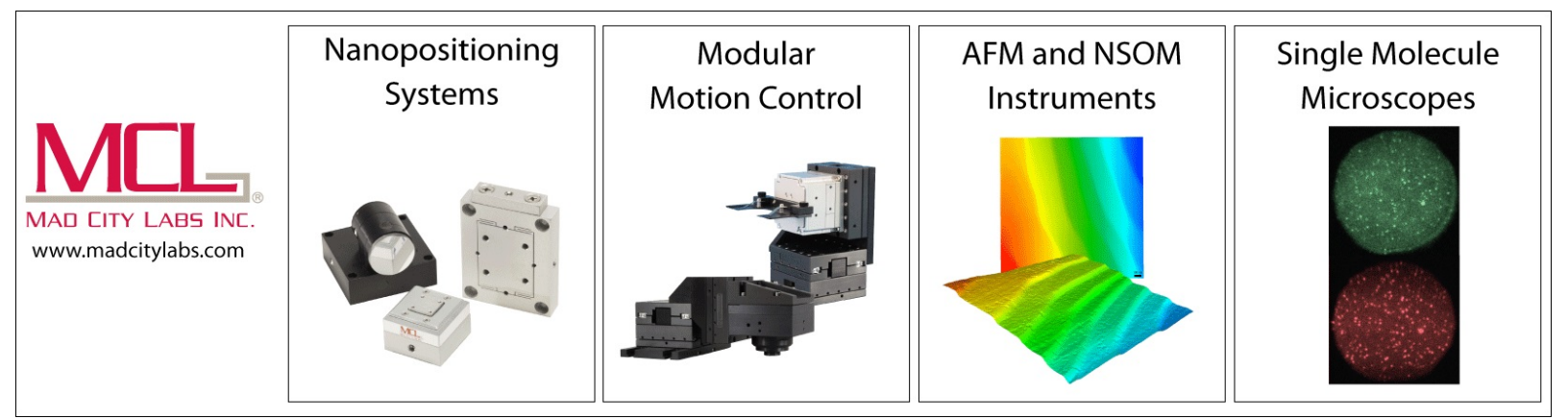




\title{
High-aspect ratio metal tips attached to atomic force microscopy cantilevers with controlled angle, length, and radius for electrostatic force microscopy
}

\author{
Lynda Cockins, Yoichi Miyahara, ${ }^{a}$ Romain Stomp, and Peter Grutter \\ Department of Physics, McGill University, 3600 Rue University, H3A 2T8 Montreal, Canada
}

(Received 9 August 2007; accepted 15 October 2007; published online 9 November 2007)

\begin{abstract}
We demonstrate a method to fabricate a high-aspect ratio metal tip attached to microfabricated cantilevers with controlled angle, length, and radius, for use in electrostatic force microscopy. A metal wire, after gluing it into a guiding slot that is cut into the cantilever, is shaped into a long, thin tip using a focused ion beam. The high-aspect ratio results in considerable reduction of the capacitive force between tip body and sample when compared to a metal coated pyramidal tip.

(C) 2007 American Institute of Physics. [DOI: 10.1063/1.2805513]
\end{abstract}

Electrostatic force microscopy (EFM) provides detection of spatially localized charges with single electron sensitivity. ${ }^{1,2}$ More recently, detection of scattering centers in mesoscopic devices ${ }^{3,4}$ as well as charge traps on semiconductor surfaces ${ }^{5-8}$ has demonstrated the great potential of this technique. EFM measurements require an electrically conductive tip in order to apply a bias voltage between tip and sample. The structure of the cantilever and, more importantly, the tip shape influence the resolution of the acquired data. $^{9-11}$ Metal coated pyramidal or conical tips are often used for EFM applications. With these tips, however, the electrostatic interaction by the body of the tip dominates over that of the tip apex, resulting in poor lateral resolution. In addition to this limitation, these kinds of coated tips are known to be fragile ${ }^{12}$ even though EFM measurements are supposed to be performed in noncontact condition.

A high-aspect ratio tip with a small radius of curvature can potentially resolve features as small as the tip diameter, and indeed examples fitting this description can be achieved by carbon nanotube tips ${ }^{13-15}$ metal wire tips, ${ }^{16,17}$ or metal deposition to create a tip. ${ }^{18}$ Metal wire tips, however, have a clear advantage for experiments at cryogenic temperatures because of more reliable electrical conduction. Akiyama et al. demonstrated a technique to fabricate high-aspect ratio metal tips glued to $\mathrm{Si}$ cantilevers using focused-ion-beam (FIB) milling. ${ }^{16}$ Although gluing tips to microfabricated cantilevers is not a new idea, the advent of FIB makes it more technically attractive and also the possibility to use any material for a tip opens up new applications in other fields.

Theoretical works by Hudlet et al. ${ }^{19}$ and Colchero et $a{ }^{20}{ }^{20}$ provide formulas for the force on the spherical apex and the conical tip body. The ratio of these two components versus cone angle of the tip (Fig. 1) emphasizes the effectiveness of reducing the cone angle so that the sample is interacting more with the apex of the tip and thus leading to an increase in resolution. Although a high-aspect ratio tip prevents the large capacitive forces of the tip body from deteriorating the resolution, such a tip must be perpendicular to the sample to fully take advantage of its capability for

${ }^{\text {a)} E l e c t r o n i c ~ m a i l: ~ m i y a h a r a @ p h y s i c s . m c g i l l . c a ~}$ reducing the background capacitive force. The cone angle of the cantilever tip is usually considered, however, this tipsample alignment $\alpha$ would act like a "half-cone angle," as depicted in Fig. 1.

In this paper, we show an improved technique to fabricate a metallic high-aspect ratio tip glued to an atomic force microscope cantilever with controlled length and, in particular, angle $\alpha$. The easy fabrication presented gives reproducible results with the entire process taking $2 \mathrm{~h}$. Our EFM results show considerable reduction in the capacitive force between tip and sample compared to a $\mathrm{Pt}$ coated $\mathrm{Si}$ cantilever-pyramidal tip.

As in most atomic force microscopes (AFM), our homebuilt cryogenic AFM (Ref. 21) has the cantilever on a $15^{\circ}$ deviation from the plane of the sample. We thus need to attach the tip on the same angle from the normal of the cantilever to correct the tip-sample angle $\alpha$. In order to predetermine $\alpha$, a FIB (FEI Dual Beam) is used to cut a triangular guiding slot into the cantilever where the metallic wire will be glued ( $5 \mathrm{nA}$ and $30 \mathrm{kV}$ ion-beam settings). The axis of the electron beam is tilted by $15^{\circ}$ with respect to the normal to the cantilever axis such that the resulting slot is tilted. This tilted slot is key to easily and reproducibly control the tip-sample misalignment. By placing the apex of the triangular slot near the middle of the existing silicon tip there will be added stability for the attached wire, however, this is not mandatory. A sufficient width for the cut pattern will cause the inside of the slot to fall out with normal handling, whereas if the cuts are too thin then the inside material will stick electrostatically to the cantilever. In the latter case, a tungsten wire (diameter of $15 \mu \mathrm{m}$ ) which is securely attached to a micromanipulator can be used for pushing out the stuck material. An effective technique for cutting the slot is to first mill most of the triangle [Fig. 2(a)], and once this is cut through, follow by milling the two sides furthest from the apex. Figures 2(a) and 2(b) show the shape of the slot that is cut into the cantilever. Cutting of the slot takes about $15 \mathrm{~min}$.

The following assembly is used to glue a wire into this fabricated slot. A linear translation stage and a $x-y$ translation stage are fastened perpendicularly to each other onto a L-shaped aluminum support. The cantilever is mounted on 


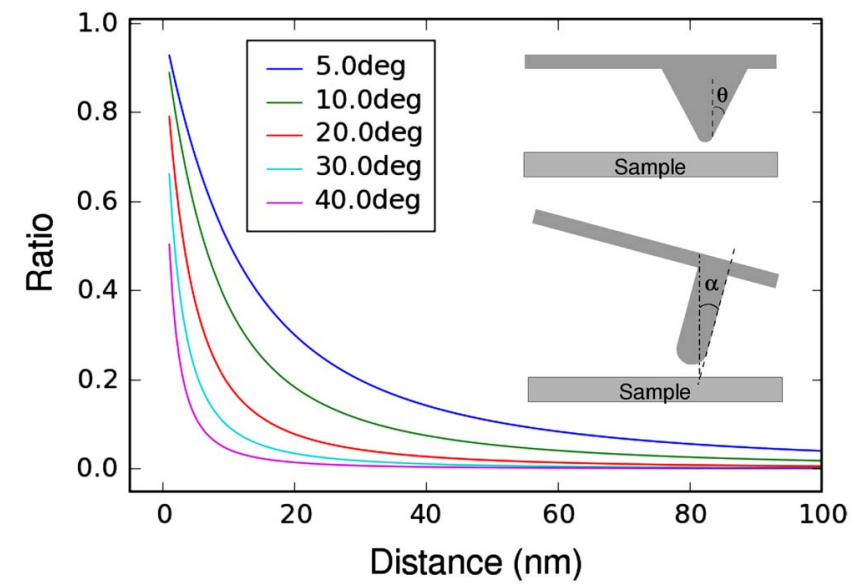

FIG. 1. (Color online) Ratio of the force on the spherical apex (radius of $10 \mathrm{~nm}$ ) of the tip compared to the total force (that acting both on the apex and conical tip body) vs tip-sample distance for different half-cone angles $\theta$. (Inset) Definition of $\theta$ and the tip-sample misalignment angle $\alpha$.

an aluminum holder with a $\mathrm{CuBe}$ leaf spring. The holder has a slanted surface so that the cantilever is pointing $15^{\circ}$ from the vertical line and is held onto the upper stage with a SmCo magnet. The cantilever assembly is mounted on the upper translation stage, which moves only in the vertical direction and is used to approach the cantilever first to a glue droplet and then to the wire. The glue and wire rest on a glass slide on top of the lower $x-y$ stage. As a precursor to the tip, we cut a $5 \mu \mathrm{m}$ diameter PtIr wire using a razor blade to a length of $\sim 1 \mathrm{~mm}$ under an optical microscope and then slide the wire over a groove cut in a glass slide. To attach the wire, the slot of the cantilever is first dipped in a small drop of silver epoxy ${ }^{22}$ and then positioned so that it straddles the wire above the groove, thereby preventing the cantilever from being glued to the glass side. If the wire is misaligned, moving the $x-y$ stage proves very effective in positioning the wire into the proper orientation as the sides of the cantilever slot can push the wire. A carbon resistor, glued onto the side

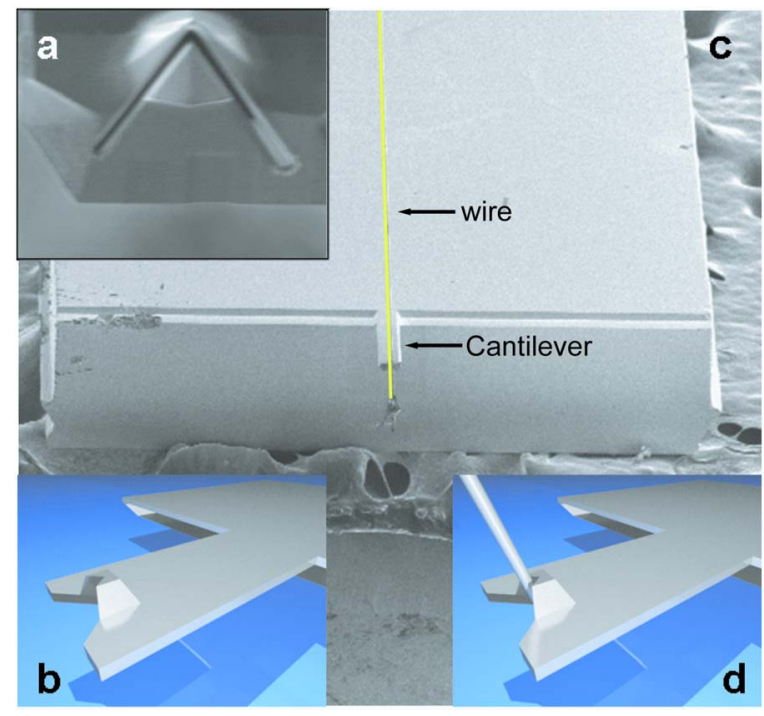

FIG. 2. (Color online) The first few steps of making these tips: The FIB cuts a slot into the cantilever (a) and falls out from handling [depicted in (b)]. A scanning electron micrograph showing the wire glued into the slot (c) [depicted in (d)].

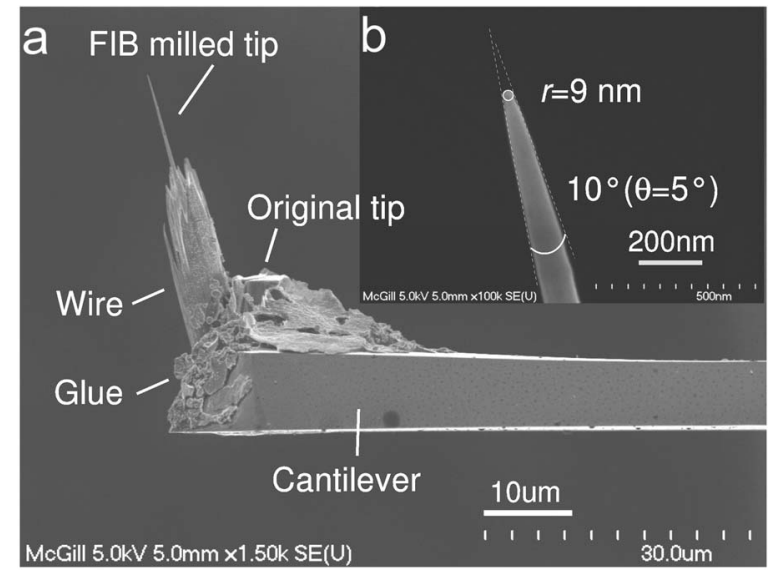

FIG. 3. A scanning electron micrograph showing the resulting tip. In (a), the wire was cut along the back side of the cantilever, the upper part of the preexisting tip was removed, and the wire was shaped after being cut at $50 \mu \mathrm{m}$ in length. A closeup of the tip is shown in (b). The radius of the apex is $9 \mathrm{~nm}$ and the half-cone angle is $5^{\circ}$.

of the cantilever holder, is used to heat and consequently cure the epoxy. This stable setup does not require any supervision to compensate the thermal drift while the glue is drying. Due to the immobilization of the wire by the glass slide and precut slot of the cantilever, a predetermined angle $\alpha$ is highly reproducible.

If starting with an uncoated cantilever a conductive layer would be deposited at this stage. Usually, however, we used coated cantilevers and found that the damage caused to the coating from the FIB is minimal because the glue covers the area of damage. Figures 2(c) and 2(d) show the cantilever with the wire glued into the slot. Finally, the cantilevers with glued wires are returned to the FIB. The wire is first cut flush along the back side of the cantilever and roughly $50 \mu \mathrm{m}$ from the surface of the cantilever. The wire is then milled further to fabricate the desired tip shape using the technique reported in Refs. 16 and 23. The shaping technique uses the FIB to successively mill smaller and smaller donut shaped patterns around the wire and usually takes less than $1 \mathrm{~h}$. Figure 3 shows a final cantilever and tip. The radius of the tip apex is $9 \mathrm{~nm}$ and the half-cone angle is $5^{\circ}$. This approach allows a reproducible fabrication of metallic tips at a predetermined angle and radius as well as high-aspect ratio determined by the FIB processing.

To demonstrate the performance of the high-aspect ratio tip, we compare the electrostatic forces measured by the high-aspect ratio tip and the coated pyramidal Si tip on evaporated gold on a mica substrate. The commerically available cantilever with pyramidal tip ${ }^{24}$ was coated with $20 \mathrm{~nm}$ thick Pt layer on top of $10 \mathrm{~nm}$ thick Ti adhesion layer. The resulting radius and half-cone angle of the coated tip are $20 \mathrm{~nm}$ and $20^{\circ}$, respectively.

The measurements were performed on atomically flat gold terraces using frequency modulation mode ${ }^{25}$ at $77 \mathrm{~K}$ at the pressure of $1 \times 10^{-4}$ Torr. For quantitative force measurements, resonance frequency shift $\Delta f$ versus distance $z$ curves were recorded at a position on the surface as a function of dc bias voltage $V_{\text {bias }}$ applied between the tip and the sample. Each $\Delta f-z$ curve was then converted into a force $F-z$ curve at each $V_{\text {bias }}$ with the method described by Sader and Jarvis. ${ }^{26}$ 


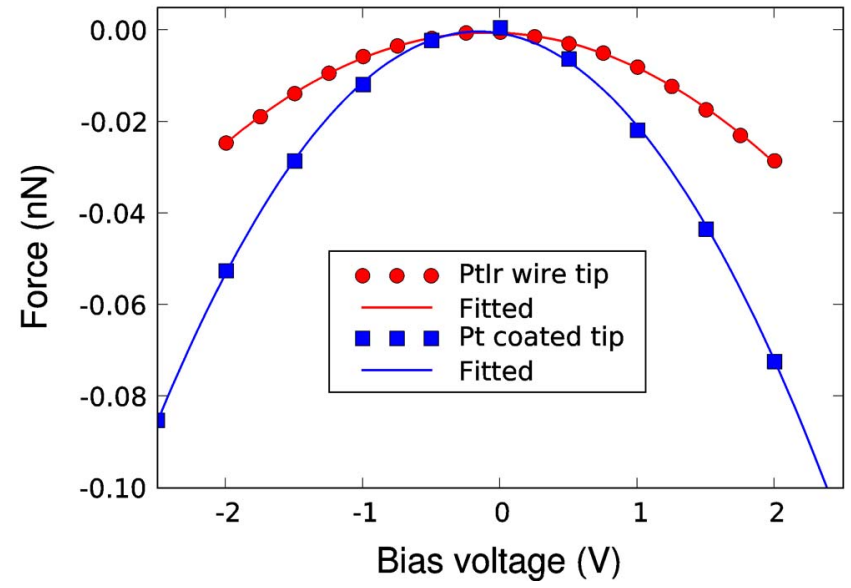

FIG. 4. (Color online) Comparison of the electrostatic forces measured by the high-aspect ratio tip and a Pt coated Si pyramidal tip at the tip-sample distance of $10 \mathrm{~nm}$.

Figure 4 shows $F-V_{\text {bias }}$ curves at $z=10 \mathrm{~nm}$ for each tip. The capacitive force given by $F=-(1 / 2) \partial C / \partial z\left(V_{\text {bias }}\right.$ $\left.-V_{\mathrm{CPD}}\right)^{2}$ accounts for the parabolic shape of these curves and its curvature gives $\partial C / \partial z$ quantitatively, where $C$ is the tipsample capacitance and $V_{\mathrm{CPD}}$ is the contact potential difference between tip and sample. Fitting of $F-V_{\text {bias }}$ curve at each tip-sample distance $z$ gives a $\partial C / \partial z-z$ curve which is shown for both tips in Fig. 5. The measured $\partial C / \partial z$ with the high-aspect ratio tip is approximately $65 \%$ less than that with the coated pyramidal tip.

In terms of the general properties of these cantilevers, we found the resonance frequency and quality factor to be within the range of the commercially available cantilever. The added mass by the attached tip and the glue is almost canceled by the cut slot and existing tip. The deviation of the angle $\alpha$ from the expected value was small (less than $0.5^{\circ}$ for a batch of approximately ten tips fabricated this way). One notable property of these tips is that they are reusable as damaged tips can be taken back to the FIB for reshaping. It is also shown in the previous work ${ }^{16}$ that the mechanical vibra-

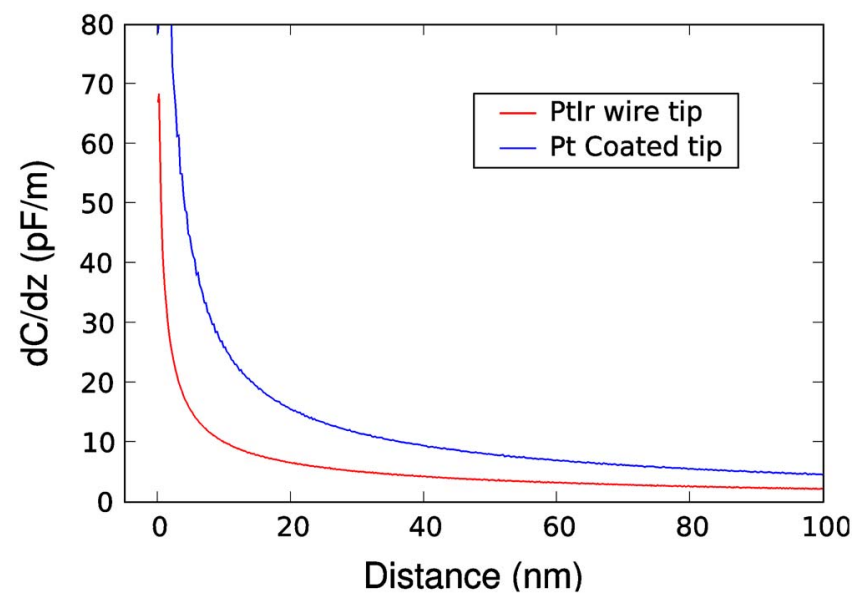

FIG. 5. (Color online) Comparison of the distance derivative of the tipsample capacitance $(\partial C / \partial z)$ by the high-aspect ratio tip and a Pt coated $\mathrm{Si}$ pyramidal tip over the tip-sample distance of $100 \mathrm{~nm}$. tion of the FIB milled metal wire tip is negligibly small and can be used for atomic resolution imaging. Such tips are thus suitable for most scanning probe microscopy (SPM) applications including EFM.

In summary, we demonstrate a method to fabricate a high-aspect ratio metal tip attached to microfabricated cantilevers. The tips take less than $2 \mathrm{~h}$ to make and allow control of all relative angles to $0.5^{\circ}$. The technique enables control of the angle, length, and radius of the tip. The controlled angle is particularly relevant in electrostatic force microscopy for reducing the electrostatic force between tip body and sample. The fabricated high-aspect tip shows 65\% smaller capacitive force when compared to a Pt coated pyramidal tip. The metal tip would also be suitable for electrical conductance measurements even at cryogenic temperature due to its robustness as a electrical conductor.

We would like to thank NSERC, RQMP, and NanoQuebec for funding. We would also like to acknowledge the technical support from FIB technicians at the Université de Montréal.

${ }^{1}$ J. E. Stern, B. D. Terris, H. J. Mamin, and D. Rugar, Appl. Phys. Lett. 53, 2717 (1988).

${ }^{2}$ C. Schonenberger and S. F. Alvarado, Phys. Rev. Lett. 65, 3162 (1990).

${ }^{3}$ J. Zhu, M. Brink, and P. L. McEuen, Appl. Phys. Lett. 87, 242102 (2005).

${ }^{4}$ P. Fallahi, A. C. Bleszynski, R. M. Westervelt, J. Huang, J. D. Walls, E. J. Heller, M. Hanson, and A. C. Gossard, Nano Lett. 5, 223 (2005).

${ }^{5}$ L. Klein and C. Williams, Appl. Phys. Lett. 79, 1828 (2001).

${ }^{6}$ L. Klein and C. Williams, Appl. Phys. Lett. 81, 4589 (2002).

${ }^{7}$ A. Dana and Y. Yamamoto, Nanotechnology 16, S125 (2005).

${ }^{8}$ R. Stomp, Y. Miyahara, S. Schaer, Q. Sun, H. Guo, P. Grutter, S. Studenikin, P. Poole, and A. Sachrajda, Phys. Rev. Lett. 94, 056802 (2005).

${ }^{9}$ H. O. Jacobs, H. F. Knapp, S. Mueller, and A. Stemmer, Ultramicroscopy 69, 39 (1997).

${ }^{10}$ H. O. Jacobs, P. Leuchtmann, O. J. Homan, and A. Stemmer, J. Appl. Phys. 84, 1168 (1998).

${ }^{11}$ S. Belaidi, P. Girard, and G. Leveque, Microelectron. Reliab. 37, 1627 (1997).

${ }^{12}$ H. O. Jacobs, H. F. Knapp, and A. Stemmer, Rev. Sci. Instrum. 70, 1756 (1999).

${ }^{13}$ S. B. Arnason, A. G. Rinzler, Q. Hudspeth, and A. F. Hebard, Appl. Phys. Lett. 75, 2842 (1999).

${ }^{14}$ N. R. Wilson and J. V. Macpherson, J. Appl. Phys. 96, 3565 (2004).

${ }^{15}$ S. D. Tzeng, C. L. Wu, Y. C. You, T. T. Chen, S. Gwo, and H. Tokumoto, Appl. Phys. Lett. 81, 5042 (2002).

${ }^{16}$ K. Akiyama, T. Eguchi, T. An, Y. Fujikawa, Y. Yamada-Takamura, T. Sakurai, and Y. Hagegawa, Rev. Sci. Instrum. 76, 033705 (2005).

${ }^{17}$ T. Eguchi, Y. Fujikawa, K. Akiyama, T. An, M. Ono, T. Hashimoto, Y. Morikawa, K. Terakura, T. Sakurai, M. G. Lagally et al., Phys. Rev. Lett. 93, 266102 (2004).

${ }^{18}$ C. Menozzi, G. C. Gazzadi, A. Alessandrini, and P. Facci, Ultramicroscopy 104, 220 (2005).

${ }^{19}$ S. Hudlet, M. Saint-Jean, C. Guthmann, and J. Berger, Eur. Phys. J. B 2 , 5 (1998).

${ }^{20}$ J. Colchero, A. Gil, and A. M. Baró, Phys. Rev. B 64, 245403 (2001).

${ }^{21}$ M. Roseman and P. Grutter, Rev. Sci. Instrum. 71, 3782 (2000).

${ }^{22}$ H20E, Epoxy Technology (http://www.epotek.com).

${ }^{23}$ M. J. Vasile, D. Grigg, J. E. Griffith, E. Fitzgerald, and P. E. Russell, J. Vac. Sci. Technol. B 9, 3569 (1991).

${ }^{24}$ NSC16 with etched Si tip, MikroMasch (http://www.stmtips.com).

${ }^{25}$ T. R. Albrecht, P. Grutter, D. Horne, and D. Ruger, J. Appl. Phys. 69, 668 (1991).

${ }^{26}$ J. E. Sader and S. P. Jarvis, Appl. Phys. Lett. 84, 1801 (2004). 